\title{
Preventing Ventilator-Associated Pneumonia in Intensive Care Unit by improved Oral Care: a Review of Randomized Control Trials
}

\author{
Ron Gershonovitch $^{1}$ (D) Noam Yarom ${ }^{1,2} \cdot$ Mordechai Findler $^{1}$
}

Accepted: 12 May 2020/Published online: 30 May 2020

(C) Springer Nature Switzerland AG 2020

\begin{abstract}
To assess the effects of various oral care methods on the incidence of VAP in patients receiving mechanical ventilation in intensive care units, an exhaustive literature search was undertaken using MEDLINE as well as a manual review of the relevant literature and citations. Eight publications were selected for this review. The primary endpoint was the incidence of VAP. Different oral care methods for preventing VAP were the subject of this review. Two studies that were reviewed in this article showed significant statistical difference between the intervention group and the control group regarding different oral care for lowering the incidence of VAP. The rest of the studies showed no significant statistical difference between the intervention group and the control group, but showed the importance of meticulous oral hygiene in those patients. Mechanically ventilated patients who suffer from poor oral hygiene are exposed to the harmful accumulation of oral plaque and the initiation of VAP. Proper oral care by qualified care givers can reduce the incidence of VAP. Proper oral care needs to be considered part of the medical treatment plan when a patient is admitted to the ICU to lower the incidence rates of VAP. Oral care treatment of ventilated patients in the intensive care units, for prevention of ventilator-associated pneumonia, is with high clinical relevance, decreasing morbidity and mortality in the ICU. We recommend that ICU medical teams will plan a protocol of oral care treatment, based on our article results, and implement it as part of the daily routine.
\end{abstract}

Keywords Ventilator associated pneumonia $\cdot$ Oral plaque $\cdot$ Intensive care unit $\cdot$ Oral hygiene $\cdot$ CHX

\section{Introduction}

The cause of death for Intensive Care Unit (ICU) patients is primarily associated with their critical illness, for which they have been admitted, and secondly, related hospital illness such as nosocomial infection. Pneumonia, an infection in one or both lungs, is the second most common nosocomial infection, affecting one of four of all critically ill patients. Ventilatorassociated pneumonia (VAP) is a nosocomial infection

This article is part of the Topical Collection on Medicine

Ron Gershonovitch

rongerso@gmail.com

Noam Yarom

noamyar@post.tau.ac.il

Mordechai Findler

findlermo@gmail.com

1 Oral Medicine Unit, Sheba Medical Center, Tel Hashomer, Israel

2 School of Dental Medicine, Tel Aviv University, Tel Aviv, Israel associated with mechanical ventilation, responsible for $86 \%$ of all nosocomial pneumonias inside the hospital [1]. This condition develops mostly after 48 hours after initiation of mechanical ventilation and endotracheal intubation. VAP increases morbidity, mortality, hospital, and ICU stay, and by this increasing healthcare cost, making it to be a serious medical condition, with attributable risk for mortality of 33-50\% [2]. In the USA alone, there are between 250,000 and 300,000 cases per year, at an incidence rate of 5-10 per 1000 admissions. Economically, the supplementary costs associated with VAP are between $\$ 5,000$ and $\$ 20,000$ per diagnosis, and treating this condition is of critical importance, along with the clinical side [1].

\section{VAP Pathophysiology}

The insertion of an endotracheal tube remains the strongest risk factor of this disease. The natural defense mechanism, such as the cough reflex, is being violated and is enabling infectious bacteria to make its way into the lower respiratory tract. Typically, there are four ways in which the 
microorganism invades the lungs: (1) A pool of secretions around the tube, (2) Micro-aspirations that usually happen when the tube is inserted, (3) A biofilm of gram-negative bacteria within the tube, and (4) the impairment of mucociliary clearance to secrete the mucus flow, especially when the patient is lying down [3]. Normal host microbial flora changes because of illness and the use of antibiotic therapy along with the management of airway protection and clearance mechanisms due to mechanical ventilation. The flora of the oropharynx is usually low in virulence. In ICU patients, the flora is overgrown by high virulence endogenic aerobic gram-negative bacilli, often causing lung infections. Colonization and infection of nosocomial bacterial pathogens can be caused by exogenous transmission from environmental sources, or they can be passed by healthcare workers treating different patients. The bacterium-enriched secretions are being "pushed" by the positive pressure ventilation, reaching moving quickly to the lower respiratory tract, initializing a lung infection [3, 4]. Immunologic factors that are damaged by the infecting organisms have a strong effect upon the initiation of this disease. Impaired phagocytosis by neutrophils, caused by the anaphylatoxin c5a from the microorganisms followed by elevation of regulatory T-cells and monocyte deactivation will predict the initiation of nosocomial infection in those patients [3].

\section{VAP Pathogens}

\section{Streptococcus pneumoniae}

A Gram-negative diplococcus pathogen who is the main cause for the early onset VAP, typically a few days after intubation.

\section{Haemophilus influenzae}

Gram negative coccobacillus, a fast-growing bacterium, induces VAP quickly after intubation.

\section{Enterobacteriaceae}

Enteric gram-negative bacilli reside mostly in the lower gastrointestinal tract.

\section{Staphylococcus aureus}

Gram-positive coccus is an important virulent factor to nosocomial infection, especially VAP.

\section{Acinetobacter Species}

Aerobic non-fermenting gram-negative bacilli are very effective in causing nosocomial infection in critically ill patients who are admitted in ICU units.

\section{Pseudomonas aeruginosa}

Aerobic, non-fermenting gram negative bacillus, the most common VAP causing pathogen, which is also resistant to antibiotics, is also the deadliest cause of VAP pathogen. [3] [4].

\section{Pathogen Profile of Oral Dental Plaque}

In the study of Sands et al. [5] different tests were taken to map the microbiology of the dental plaque residing in the oral cavity of VAP patients. Five different bacterial phyla were studied from the specimens of 13 patients. Forty genres, six of which were not part of the normal oral plaque community and the following four major species were documented in 38 dental plaque specimens: Streptococcus pseudopneumoniae (66 \%), Enterococcus faecalis (37\%), and Escherichia coli and Shigella flexneri (32\%).From the study (Sands et al.), it was concluded that there is a difference in the profile of the pathogen in the beginning of the intubation versus the end of it. Another interesting evidence extracted from this article, is that Pseudomonas aeruginosa was not identified in the dental plaque of these patients, although past studies demonstrated this bacterial species is a predominant colonizer in the dental plaque of ventilated, critically ill patients, as well as a major cause of VAP (Parker et al 2008; Raad et al 2011 Tarquinio et al 2014). Information about the profile of the pathogens involved in the oral plaque of these patients will help create intervention strategies to offset the incidence of VAP [5].

\section{Common Methods for Prevention of VAP}

Strategies for the prevention of ventilator-associated pneumonia includes the following: prophylactic antibiotic administration, sedation interruption, head-end elevation, limitation of ventilation times, performing endotracheal suctioning, avoiding gastric overdistention, draining ventilator tube condensate, Kinetic bed therapy, and other factors that deals with the ventilator machine itself $[1,2]$.

\section{Oral Care for Prevention of VAP}

Comprehensive oral care is considered a vital component in the prevention of VAP. The most common measure is suction of the oropharyngeal secretions that is "rich" with bacterial load. Other methods of oral care for preventing ventilatorassociated pneumonia that are commonly used for ventilated patients include the following: chlorhexidine as monotherapy in different concentrations of $0.12 \%, 0.2 \%, 1 \%$, and $2 \%$ that can be applicate as oral rinse, gel, or foam and mechanical debridement in the form of toothbrushing manual or electric, as monotherapy or in conjunction with chlorhexidine. Other 
decontamination agent includes povidone-iodine $10 \%$, and other forms of mouthwash [1, 2, 6].

\section{Objectives}

The objective of this study is to assess the beneficial effects of various oral care methods on the incidence of VAP in critically ill patients receiving mechanical ventilation in intensive care units in hospitals.

\section{Methods and Materials}

\section{Search Methods and Selection Criteria}

An exhaustive literature search was undertaken using MEDLINE as well as a manual review of the relevant literature and citations. The cited articles were published in medical literature from 2008 to 2018 regarding VAP and oral care. Study selection was made independently by the three authors (RG, NY, and MF).

To find and filter unrelated articles, we used keywords from the medical subject headings (mesh): (((Ventilator Associated Pneumonia) OR (VAP)) and ((Oral Care) or (Oral Hygiene) or (Oral Rinse) or (Mouthwash)) and ((Intensive Care Unit) or $(\mathrm{ICU}))$ ). Selection of the studies started with search in MEDLINE using the keyword "mesh."

When the query was entered, the first result produced 235 articles containing the word mesh. The first filter applied was only for full-text articles which resulted in eight articles being excluded. The next filters were as follows: The type of infection (ventilator associated pneumonia), article type (randomized control trials), age (adult aged 19+), treatment agent (oral care decontamination), type of patients (ventilated patient in the ICU's), and studies which were concluded in the last 10 years. After using those filters, 219 articles were excluded, and eight studies were included in the qualitative synthesis. After the selection of the articles, results were extracted and analyzed. Discussion about the results and their importance to the study question was the final part of this review.

\section{Results}

Searching studies for this review came up with 235 references in MEDLINE (Fig. 1). After reviewing the inclusion criteria, eight publications were selected for this review.

\section{Endpoints}

The primary endpoint in all the studies was the incidence of VAP. Secondary endpoints that were measured were mortality rate [7, 8, 11-13], length of ICU stay [8, 11-13], antibiotics free days [7], mechanical ventilation free days [7, 11],common microorganism colonization [9, 11, 13], clinical periodontal measurements [13], and rate of tracheobronchitis or acute respiratory stress disorder [10] . In this study our only interest was in the primary endpoint that was the incidence of VAP and ignored the rest of the data.

Fig. 1 Article flow chart
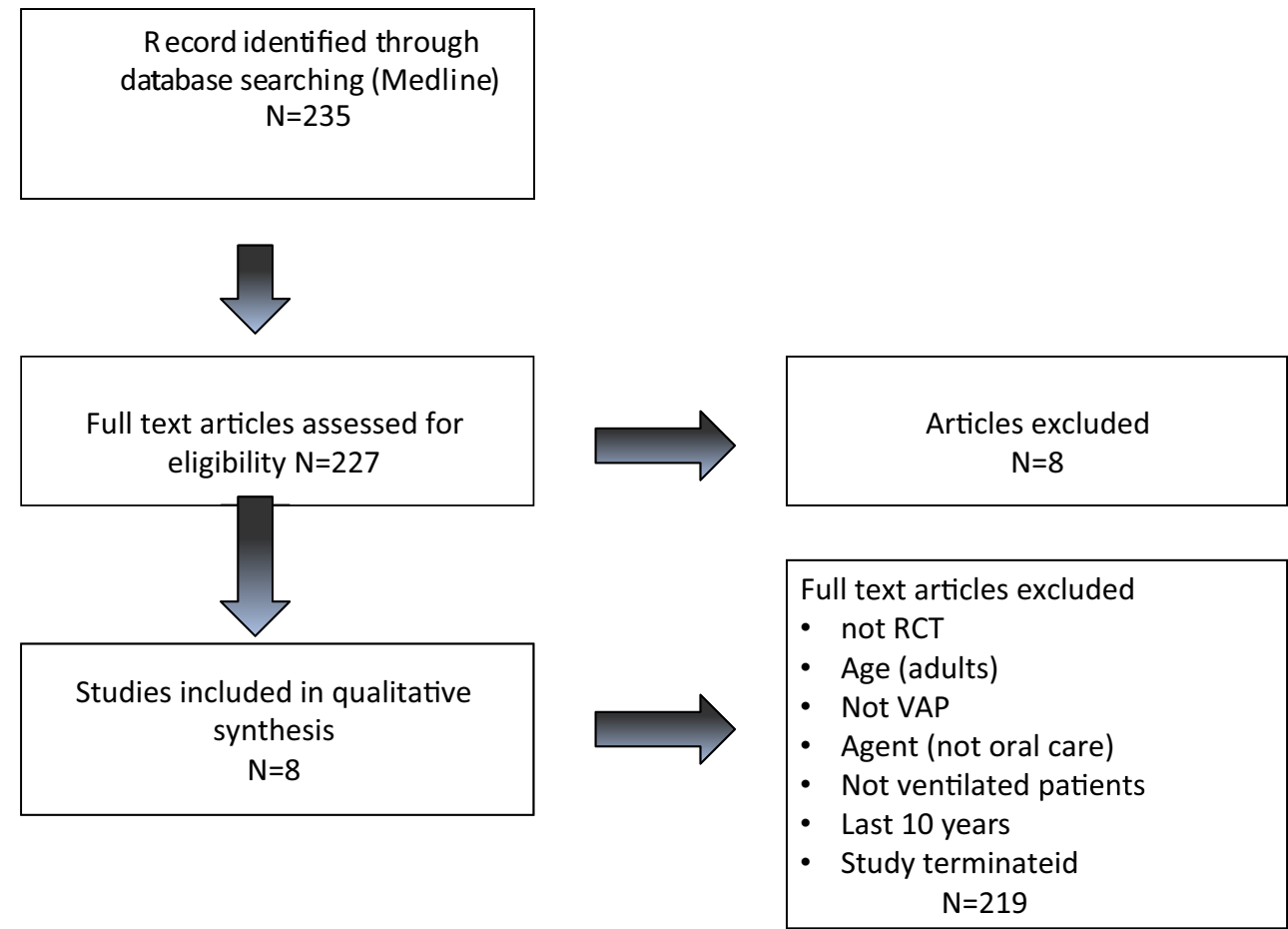


\section{Differentiation of Oral Prevention Methods}

Different oral care methods for preventing VAP were the subject of this review. The main oral agent in the intervention groups was presented accordingly: $25 \%$ of all reported methods demonstrated the use of chlorhexidine $0.12 \%$ rinse, $37.5 \%$ were with chlorhexidine $0.2 \%$ rinse, and $12.5 \%$ were with chlorhexidine $2 \%$ rinse. The others were treated with $12.5 \%$ povidone-iodine and $12.5 \%$ mechanical intervention by use of a toothbrush. Of those treated, $87.5 \%$ of the cases were initialized with suction of the oropharyngeal secretions, when only in the povidone-iodine intervention, suction of the secretions did not happen. A combination of two main interventions occurred when chlorhexidine and a toothbrush combined in $62.5 \%$ of the cases and a toothbrush with sodium bicarbonate mouthwash in $12.5 \%$ of the cases. In the control group, $25 \%$ of the groups treated with CHX (i.e., chlorhexidine) $0.2 \%$ and $37.5 \%$ with CHX $0.12 \%$. The rest of the control groups were given a placebo gel, sterile water, and saline.

\section{Analysis of the Results}

\section{CHX 2\% + Mechanical Toothbrush}

Analysis of the results demonstrated the following conclusions. In the study of Zand et al. [11], 114 patients were allocated into two groups. Both the intervention and control groups received oral care in the way of suctioning the oropharyngeal secretions as well as brushing the oral cavity and teeth. The intervention group was treated with $\mathrm{CHX} 2 \%$ and the control group with CHX $0.2 \%$. This study showed a significant difference regarding the incidence of VAP between the two study groups although there was no significant difference between the two groups regarding mean ventilation days, or 28 days following ventilation therapy. In the control group the incidence of VAP was $22.8 \%$, and in the intervention group only $5.3 \%$. This result was statistically significant $(P$ value $=$ $0.007)$.

\section{CHX 0.12\% + Mechanical Toothbrush}

In the study of Lacerda Vidal et al. [12], 213 patients were allocated into two groups: Control $(n=108)$ and study group $(n=105)$. They received oral care with suction oropharyngeal secretions and a mouth swab with CHX $0.12 \%$. The intervention group was treated with a thorough toothbrushing and cleansing of the oral cavity. In this study, the control group's incidence of VAP was higher than the intervention group (25.9\% and $16.19 \%$, respectively) although the statistical difference was not significant $(P$ value $=0.084)$.

In the study of Lorente et al. [8], the authors compared an intervention group $(N=217)$ who were given oral care with
CHX $0.12 \%$ with toothbrushing and suctioning of oropharyngeal secretions with a control group $(N=219)$ with suctioning of oropharyngeal secretions and rinsing and swabbing with CHX $0.12 \%$, without toothbrushing. There was no significant difference between the groups regarding the incidence of VAP.

In the case study of Pobo et al. [7], where the intervention group contained 74 patients and the control group contained 73 patients, electric toothbrushing was the mechanical agent rather than a manual one. A difference was not found between the study and the control group. Additionally, the control group showed lower VAP incidence relative to the study group (20.3\% and $24.7 \%$, respectively).

\section{CHX $0.2 \%$}

In a study conducted by Ozcaka et al. [13] 29 patients in the intervention group, and 32 patients in the control group, were the target population. Routine oropharyngeal suction of oral secretions was conducted both in the control and intervention groups. The difference was the use of mouth swab and oral rinse with $\mathrm{CHX} 0.2 \%$ in the intervention group. The result from this study demonstrated a higher incidence of VAP in the control group relative to the intervention group (study, 41.4\%; control, $68.8 \%)$, in a statistically significant way $(P$ value $=0.03$ ).

\section{CHX 0.2\% + Mechanical Toothbrush}

In the study of Chacko et al. [6] a comparison between the intervention group consisted of 104 patients who were given oral care in the form of suctioning of the oropharyngeal secretions, a mouth swab containing CHX $0.2 \%$ and an oral cavity cleaning with manual toothbrush. A control group of $102 \mathrm{pa}-$ tients were given the standard oral care of a mouth swab cleaning with CHX $0.2 \%$. Inclusion criteria in this study is mechanical ventilation of ad list 4-6 h. The authors did not find strong statistical differences between the groups ( $p$ value $=0.82$ ), suggesting that toothbrushing and suctioning of the oropharyngeal secretions did not have any more effect to standard oral care.

\section{CHX 0.12\%/Sodium Bicarbonate + Mechanical Toothbrush}

In the study of Berry et al. [9], the author noted that using different kinds of mouth rinses (CHX $0.12 \%$, sodium bicarbonate, and saline) along with mechanical toothbrushing did not affect the incidence of VAP in any significant way. It was concluded that brushing had the most effect on lowering the incidence of VAP. 


\section{Povodine-lodine $10 \%$}

In the study of Seguin et al. [10], with 150 patients consisting of 78 patients in the intervention group and 72 in the control group, the oral agent was iodine-povidone $10 \%$ compared with placebo gel with sterile water. The authors concluded there was no significant difference in the incidence of VAP to the intervention group ( $p$ value $=0.69$ ). This study showed that povidone-iodine lowered the oropharyngeal colonization but not the endotracheal colonization, and the use of povidone-iodine and this did not change the incidence of VAP in the study group. Also, it was demonstrated that povidone-iodine can cause an acute respiratory disorder and is not recommended to be used as a decontamination agent.

\section{Discussion}

The proper oral care treatment of ventilated patients in the intensive care units for prevention of ventilator-associated pneumonia became an important line of defense for the eradication of antibiotic-resistant microorganisms, decreasing morbidity and mortality among patients in the ICU as well as the lowering of health care costs. In this review, different methods of oral care were given to ventilated patients in various intensive care units by the care takers in the units. Randomized control trials which test different kind of oral care are presented in Table 1.

Two studies that were reviewed in this article showed significant statistical difference between the intervention group and the control group regarding different oral care for prevention of VAP. Zand et al. [11] gave different concentrations of chlorhexidine orally to the intervention group and the control group $(2 \%, 0.2 \%)$, respectively, and found that the incidence of VAP decreased dramatically, proving that not only the type of oral care while on a ventilator is important but also the concentration amount of the agent has a critical role for decreasing morbidity. In the study of Ozcaka et al. [13] significant statistical differences occurred between the intervention group and the control group. The intervention group was treated with mouth swab of chlorohexidine $0.2 \%$, and in this study, there was a strong connection for the number of ventilated days of the incidence of VAP. There was more effectivity in providing a mouth swab to a patient than regular rinse because it reached and penetrated more tissue. There is a noticeable difference in the number of times a day an oral CHX swab was given. It was also concluded that lowering the offset of VAP is in strong connection with a more experienced and professional care takers responsible to the oral care that was given.

In three other studies $[7,8,12]$, presenting an intervention group given mechanical debridement (manual or electric toothbrush) along with the antiseptic agent, there was no significant difference between the intervention and the control group. In those studies, it was proven that adding a toothbrush (electric or manual) did not contribute more than the CHX already did. Also, it was concluded that the chemical effect of CHX is effective for "moving" microorganisms and does not affect the oral plaque that is built up as a biofilm. The effectivity of the CHX is only noticeable after mechanical debridement by breaking the thick biofilm that is the reservoir for the respiratory pathogens. Another important conclusion from the studies was noting that the presenting patient admitted to the ICU had poor oral health, which contributed to accumulation of dental plaque, which later was a reservoir for respiratory pathogens and later aspirated into the lungs causing VAP.

In contrast to those studies, Barry et al. [9] tested if there is a difference between different oral rinses in decreasing the incidence of VAP and found no statistical significance. The author concluded that the mechanical debridement of oral plaque (toothbrushing) had the most effect decreasing the incidence of VAP.

Chacko et al. [6] did not find significant differences between the intervention group receiving mechanical (toothbrushing) and chemical (mouth swab of CHX 0.2\%) debridement and the control group receiving only mechanical debridement (toothbrushing). The authors discovered that the risk of having VAP while on mechanical ventilation increased everyday by 1.3 , suggesting that the method of the oral care did not had the strongest effect on the incidence of VAP, but the number of ventilation days. In this study, the incidence of VAP relatively to general ICU population was low but it was concluded that a potential caregiver bias influenced on this study.

In the study of Seguin et al. [10], again no statistical difference was found between the intervention and the control group regarding the use of povidone-iodine as the oral antiseptic. The author had found that the more ventilation days, the higher dental plaque accumulated in the oral cavity and there was a connection between dental plaque pathogens and respiratory pathogens responsible for causing VAP.

We concluded from this review that if a patient is admitted to the ICU who suffers from poor oral hygiene, they contribute to the harmful accumulation of oral plaque and this is also responsible for the pathogens which eventually cause the initiation of VAP and more ventilation days. This data confirms that the incidence of VAP influenced more by the number of ventilation days. Lowering dental plaque by proper oral care will lower the incidence of VAP.

Because of that conclusion, it is extremely important for ventilated patients in the ICU to be given proper oral care by qualified care givers for the prevention of VAP. Administration of chlorohexidine in different concentrations alongside the suction of oropharyngeal secretions and mechanical debridement gave good results in the lowering of the accumulation of the pathogens responsible for the 


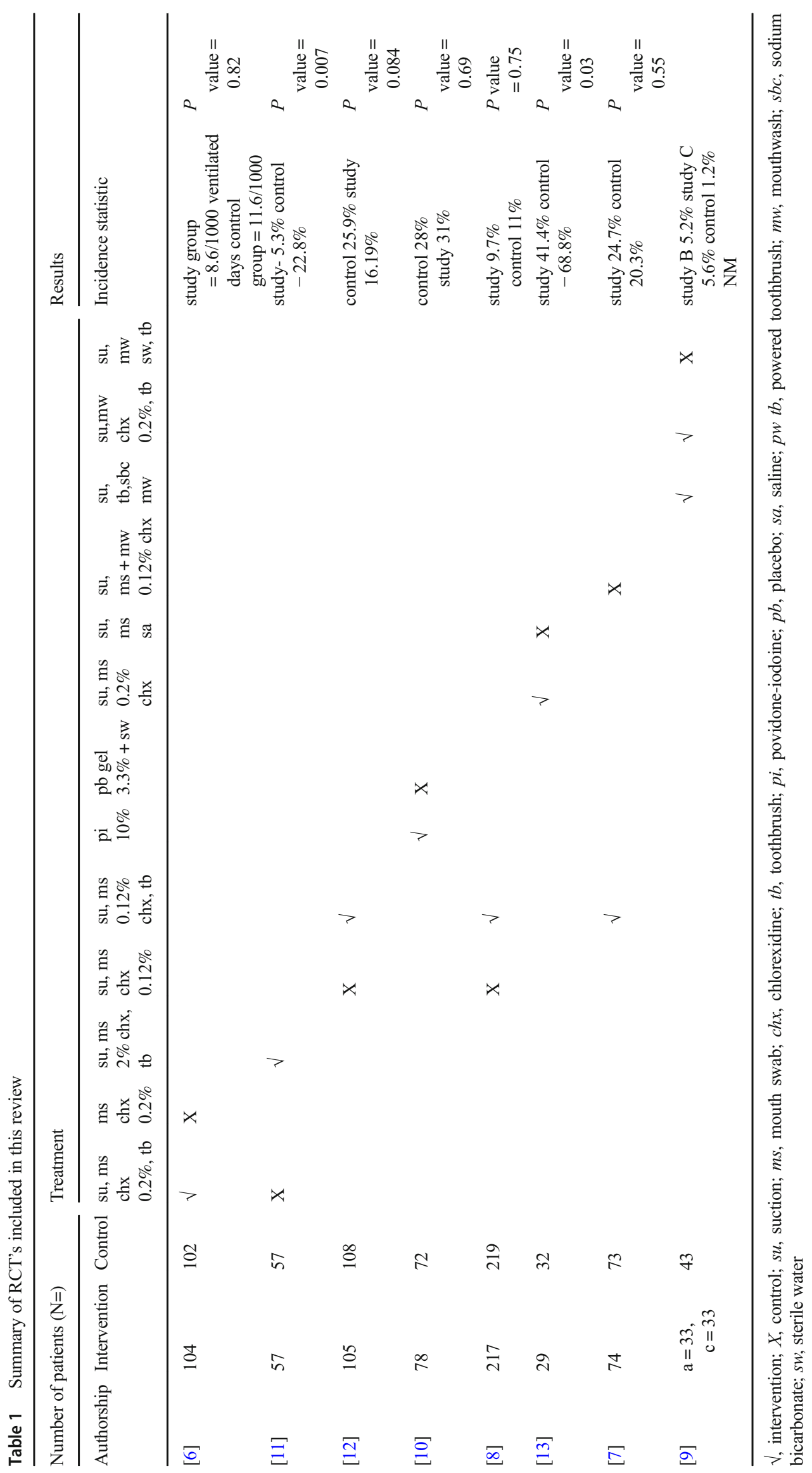


initiation of VAP. Also, it was concluded from this review that the more professional and experienced caretakers providing proper oral care protocols, the lower incidence of VAP will occur.

In conclusion, proper oral care needs to be considered part of the medical treatment plan when a patient is admitted to the ICU to lower the incidence rates of VAP.

\section{Compliance with Ethical Standards}

Conflict of Interest Author Ron Gershonovitch declares that he has no conflict of interest. Author Noam Yarom declares that he has no conflict of interest. Author Mordechai Findler declares that he has no conflict of interest.

We wish to confirm that there are no known conflicts of interest associated with this publication and there has been no significant financial support for this work that could have influenced its outcome.

Funding Information The work was supported by the oral medicine unit, sheba medical center, tel hashomer, israel.

Ethical Approval All procedures performed in studies involving human participants were in accordance with the ethical standards of the institutional and/or national research committee and with the 1964 Helsinki declaration and its later amendments or comparable ethical standards.

Informed Consent For this type of study, formal consent is not required.

\section{References}

1. Koenig SM, Truwit JD. Ventilator-associated pneumonia: diagnosis, treatment, and prevention. Clin Microbiol Rev. 2006;19(4): $637-57$.

2. Villar CC, Pannuti CM, Nery DM, Morillo CM, Carmona MJ, Romito GA. Effectiveness of intraoral Chlorhexidine protocols in the prevention of ventilator-associated pneumonia: meta-analysis and systematic review. Respir Care. 2016;61(9):1245-59.

3. Kalanuria AA, Ziai W, Mirski M. Ventilator-associated pneumonia in the ICU. Crit Care. 2014;18(2):208. https://doi.org/10.1186/ cc13775 Review. Erratum in: Crit Care. 2016; 20:29.
4. Park DR. The microbiology of ventilator-associated pneumonia. Respir Care. 2005;50(6):742-63.

5. Sands KM, Twigg JA, Lewis MAO, Wise MP, Marchesi JR, Smith A, et al. Microbial profiling of dental plaque from mechanically ventilated patients. J Med Microbiol. 2016;65(Pt 2):147-59.

6. Chacko R, Rajan A, Lionel P, Thilagavathi M, Yadav B, Premkumar J. Oral decontamination techniques and ventilatorassociated pneumonia. Br J Nurs. 2017;26(11):594-9.

7. Pobo A, Lisboa T, Rodriguez A, Sole R, Magret M, Trefler S, et al. A randomized trial of dental brushing for preventing ventilatorassociated pneumonia. Chest. 2009;136(2):433-9.

8. Lorente L, Lecuona M, Jiménez A, Palmero S, Pastor E, Lafuente $\mathrm{N}$, et al. Ventilator-associated pneumonia with or without toothbrushing: a randomized controlled trial. Eur J Clin Microbiol Infect Dis. 2012;31(10):2621-9.

9. Berry AM, Davidson PM. Master's J, Rolls K, Ollerton R. Effects of three approaches to standardized oral hygiene to reduce bacterial colonization and ventilator associated pneumonia in mechanically ventilated patients: a randomized control trial. Int J Nurs Stud. 2011;48(6):681-8.

10. Seguin P, Laviolle B, Dahyot-Fizelier C, Dumont R, Veber B, Gergaud S, et al. Effect of oropharyngeal povidone-iodine preventive oral care on ventilator-associated pneumonia in severely braininjured or cerebral hemorrhage patients: a multicenter, randomized controlled trial. Crit Care Med. 2014;42(1):1-8.

11. Zand F, Zahed L, Mansouri P, Dehghanrad F, Bahrani M, Ghorbani $\mathrm{M}$. The effects of oral rinse with $0.2 \%$ and $2 \%$ chlorhexidine on oropharyngeal colonization and ventilator associated pneumonia in adults' intensive care units. J Crit Care. 2017;40:318-22.

12. de Lacerda Vidal CF, Vidal AK, Monteiro JG Jr, Cavalcanti A, Henriques APC, Oliveira M, et al. Impact of oral hygiene involving toothbrushing versus chlorhexidine in the prevention of ventilatorassociated pneumonia: a randomized study. BMC Infect Dis. 2017;17(1):112.

13. Özçaka Ö, Bașoğlu OK, Buduneli N, Tașbakan MS, Bacakoğlu F, Kinane DF. Chlorhexidine decreases the risk of ventilatorassociated pneumonia in intensive care unit patients: a randomized clinical trial. J Periodontal Res. 2012;47(5):584-92.

Publisher's Note Springer Nature remains neutral with regard to jurisdictional claims in published maps and institutional affiliations. 